\title{
The Physics of EARTHQUAKES
}

$\mathbf{T}$ he recent earthquakes in Taiwan, Turkey, and India tragically demonstrate the abruptness with which earthquakes occur and the devastation that often accompanies them. Scientists, emergency officials, and the public are greatly interested in earthquakes-sudden fractures in Earth's crust followed by ground shaking-and have many questions about them. For example, When do earthquakes occur? More precisely, what long-term processes and short-term triggers produce earthquakes? Although plate tectonics has provided a successful framework for understanding the long-term processes, the short-term triggers remain obscure, making earthquakes unpredictable. An equally important question and a fundamental challenge to the science of geophysics is, What happens during an earthquake? That is, What are the forces and motions during a seismic event? The answer to this question has practical consequences for mitigating the effects of the expected ground motion.

One of the most challenging aspects of studying earthquakes is obtaining observational constraints. Most earthquakes occur at depths down to $50 \mathrm{~km}$, but some as deep as $670 \mathrm{~km}$ have been observed in certain regions. Seismologists have never directly observed ruptures occurring in Earth's interior. Instead, they rely on the information gleaned from the few available types of data, the most important of which is the record of seismic waves. During an earthquake, sudden crustal motion excites elastic waves that travel through Earth and are observable at seismic stations on the surface. These waves carry information about the movements at the earthquake's source, but the complex structure of Earth between the source and the receiver often complicates extracting the information from the signal. Despite this difficulty, researchers have learned much from seismic records and can determine the detailed rupture history of many recent seismic events.

Geological observations of exhumed faults (old faults formed at depths of about $5-10 \mathrm{~km}$ and brought to the surface by long-term uplift), geodetic measurements of crustal motion, heat-flow measurements, and laboratoryanalog experiments have all significantly added to our information base. Even a simple catalog of when and where earthquakes occur helps to identify patterns that may point to common causes or to interactions between events (see PHYSICS TODAY, April 2000, page 59).

Earthquake physicists attempt to link the available observations to the processes occurring in Earth's deep

HIROO KANAMORI is the Jobn E. and Hazel S. Smits Professor of Geo. physics at the Seismological Laboratory, California Institute of Tecbnolo gy in Pasadena. EMILY E. BRodsky is currently a National Science Foundation Geoscience Postdoctoral Fellow at the University of Oregon in Eugene. interior to help them interpret the types of data just described. A few approaches or paradigms are commonly used to create these links. For example, plate tectonics links geodetic observations to the stresses that generate earthquakes over geological time scales. Models of frictional behavior link laboratory-analog and certain seismic waveform features to the stress changes occurring during an earthquake. In this review, we cover a few of the most commonly used approaches in earthquake physics. ${ }^{1}$ Some more recent and speculative ideas provide an insight into possibly fertile future directions of research. Throughout, we are driven by the question, What happens during an earthquake?

\section{Long-term processes}

The forces generated in Earth's crust are typically described in terms of the shear stress and the shear strain. The shear stress is the force per unit area applied tangent to a plane. The shear strain is a dimensionless quantity that describes the distortion of a body in response to a shear stress; shear strain is defined in box 1 (page 36). In this article we are concerned with shear forces and their effects, so for brevity, we do not use "shear" when discussing stresses and strains.

Long-term loading has traditionally been measured by geodetic and geological methods. Recent progress in space-based geodesy, made possible by the global positioning system and satellite interferometry, now provide us with a clear pattern of crustal movement and strain accumulation. Figure 1 shows recent geodetic measurements in California. The relative plate motion determined from these data is about $2-7 \mathrm{~cm} /$ year, which translates into a strain accumulation rate of approximately $3 \times 10^{-7} / y$ along plate boundaries. The strain also accumulates in plate interiors, but at a much slower rate of about $3 \times 10^{-8 / y}$ or less. Since the rigidity of the crustal rocks is about $3 \times 10^{4} \mathrm{MPa}$, this corresponds to a stress accumulation rate of $10^{-2} \mathrm{MPa} / \mathrm{y}$ along plate boundaries, an order of magnitude less in plate interiors.

When the stress at a point in the crust exceeds a critical value, called the local strength, a sudden failure occurs. The plane along which failure occurs is called the fault plane and the point where failure initiates is called the focus. Typically, there is a sudden displacement of the crust at the fault plane following the failure, and elastic waves are radiated. This is an earthquake. For most earthquakes, the displacement occurs at an existing geological fault, that is, a plane that is already weak.

The strain change, or coseismic strain drop, associated with large earthquakes has been estimated using geodetic and seismological methods. It ranges roughly from $3 \times 10^{-5}$ to $3 \times 10^{-4}$, as demonstrated by Chuji Tsuboi's pioneering analysis of the 1927 Tango, Japan, earthquake. $^{2}$ The corresponding change in stress, called the 


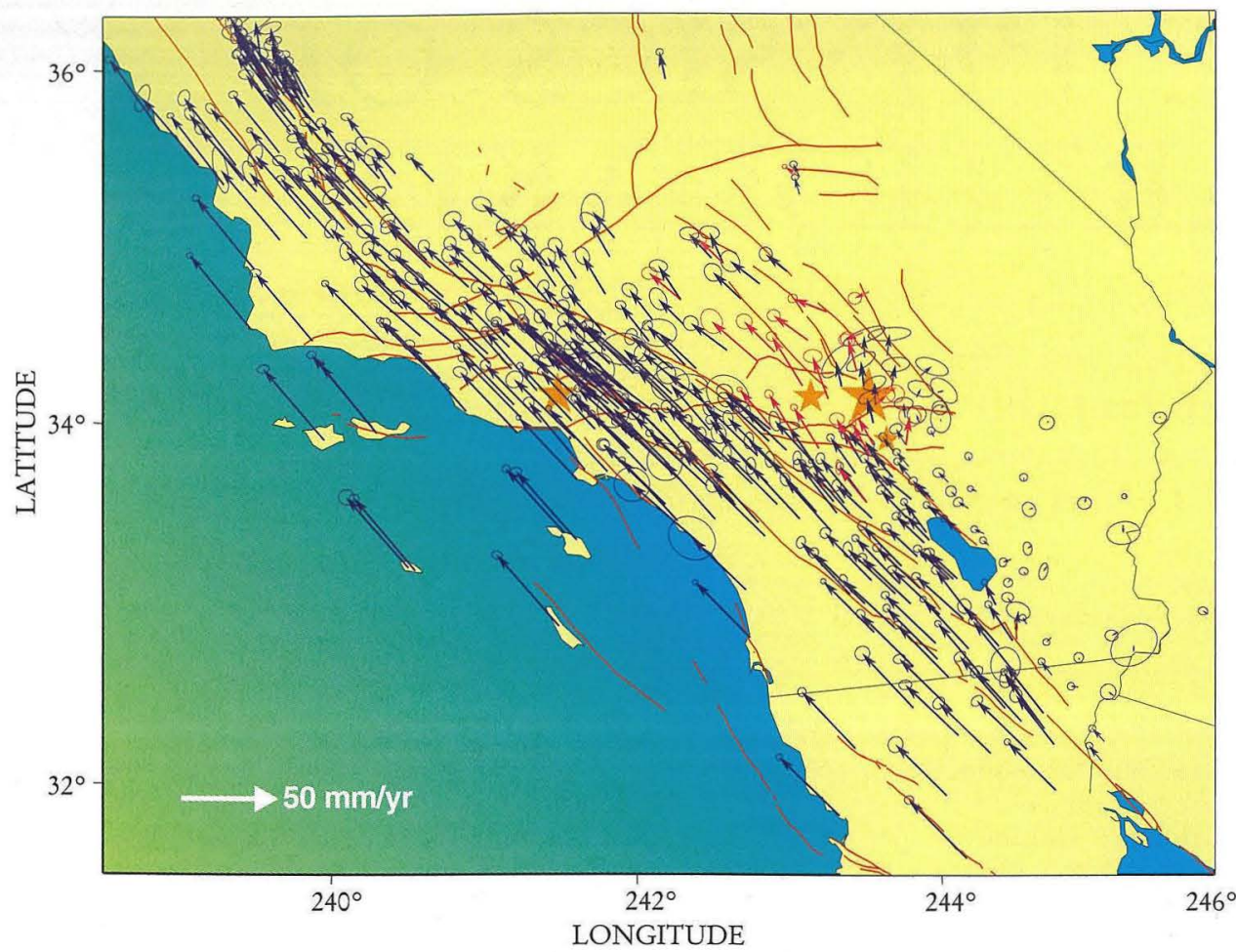

FIGURE 1. VELOCITY vectors determined by the global positioning system and other space-based methods show the plate motion in southern California. The deformations associated with recent earthquakes (orange stars) have been removed from the map in order to isolate the long-term average motion. Red arrows are the site velocities possibly changed after the Landers and Northridge earthquakes. Error ellipses correspond to $95 \%$ confidence levels. The figure is part of the Southern California Earthquake Center's website, http://www.scecdc.scec. org/group_e/release.v2.

is presented in box 1 .

In general, fault motion does not occur smoothly, but rather in a stop-and-go fashion, called stick-slip. The dynamic effects leading to

static stress drop, is about $1-10 \mathrm{MPa}$, which is at least an order of magnitude smaller than the several hundred $\mathrm{MPa}$ needed to break intact rocks in the laboratory.

Dividing the coseismic strain drop by the strain accumulation rate suggests that the repeat times of major earthquakes at a given location are about $100-1000$ years on plate boundaries, and about 1000-10000 years within plates. These values agree with what has been observed at many plate boundaries and interiors.

Figure 2a schematically shows the development over time of stresses that generate earthquakes. Although the basic process is well understood and accurately measured, its details are quite complex. For example, the stress accumulation rate is not uniform over time. A large earthquake on a segment of a fault changes the stress on the adjacent segments, either statically or dynamically, and accelerates or decelerates seismic activity, depending on the fault geometry. The strength of the crust is not constant over time either. Migrating fluids may weaken Earth's crust significantly, altering the times at which earthquakes occur. The stress drop during earthquakes may also vary from event to event. These complicating factors and their effect on the intervals between earthquakes are illustrated in figure $2 \mathrm{~b}$. The overall long-term process is regular, but considerable temporal fluctuations of seismicity occur, making accurate prediction of earthquakes extremely difficult.

\section{Short-term processes and friction}

Earthquake fault motion can be viewed as frictional sliding on a fault plane. The friction changes as a function of slip (relative displacement of the two sides of the fault plane), velocity, and history of contact. Thus, frictional stress controls seismic motion. An earthquake can occur only if friction decreases rapidly with slip, a process referred to as slip weakening. If friction increases with slip, or does not drop rapidly enough, slip motion either stops or occurs gradually. We treat only the slip-weakening case in this article. A simple model for seismic motion stick-slip behavior have been studied extensively. ${ }^{3}$ (See the article "Rubbing and Scrubbing" by Georg Hähner and Nicholas Spencer in PHYSICS TODAY, September 1998, page 22.) In general, geophysical stick-slip occurs in the following sequence: The tectonic loading stress accumulates until it exceeds the frictional stress; sliding begins; the loading stress drops below that of friction; the fault motion stops; and the process repeats. "Sticking" requires that the loading stress be less than the frictional stress. This can occur in the midst of an earthquake because of geometrical and compositional heterogeneity within the fault plane. More sophisticated models include velocityand history-dependent friction, and predict stick-slip due to purely dynamic effects.

Both small-scale spatial variations in frictional properties and dynamic effects control the physics of seismic slip on a microscopic level. However, earthquakes are inherently large events with slip displacements as large as $10 \mathrm{~m}$ and particle velocities up to $3 \mathrm{~m} / \mathrm{s}$. It is important that earthquake physicists develop theoretical tools to understand how microscopic processes produce the observed macroscopic behavior.

Seismic waves radiated during earthquakes provide evidence that the properties on a fault plane are indeed complex. Seismologists can use data from modern broadband seismometers to invert observed waveforms and obtain the distribution of slip on a fault plane. Figure 3 shows the total slip on the fault plane that occurred during the 1992 Landers, California, earthquake. (See PHYSICS TODAY, September 1993, page 17.) Such maps demonstrate that small-scale processes are inherently part of the earthquake process.

The irregular distribution of slip reflects both the complexity of dynamic frictional stress and the heterogeneity of local strength on the fault surface. Structure on finer scales than shown in the figure is likely to be present, but must be omitted from the inversion because of bandwidth limitations. The highest-frequency waves, which sample the 


\section{Box 1. Seismological Variables}

$T^{\text {the }}$ material of Earth's crust is constantly subjected to forces and it deforms in response to them. The figure at the top illustrates a slab of crustal rock with a simplified geometry. Equal but oppositely directed forces act tangent to the red and blue planes on the boundary of the slab. The stress is defined as the magnitude of the force divided by the area of the planes. Because of the stress, the slab is deformed from the cuboid shape it would have in the absence of applied forces. The figure shows that the red plane is displaced downward a distance $y$ relative to the blue plane, and that the separation distance of the two planes is $x$. The strain in the slab is, by definition, $y / x$. The strain is proportional to the stress with the constant of proportionality depending on the material from which the slab is made. The ratio of stress to strain is called the rigidity of the slab material, $\mu$. When the slab is infinitesimal, the preceding discussion defines the stress and strain at the point enclosed by the slab.

In Earth's crust there are planes that can support only relatively low stresses before rupturing. These weak planes are called fault planes. In a simple model, an earthquake is precipitated when the stress on a fault plane exceeds the static frictional stress, $\sigma_{0}$. Plates on either side of the fault experience a relative displacement, or slip, $D$ over an area $S$, as illustrated in the middle figure. The displacement may be as great as $10 \mathrm{~m}$, but the linear dimension of a fault in the direction of the displacement is typically tens of kilometers so that the contact area is essentially unchanged as the plates move. As sliding commences, the frictional stress drops to a lower kinetic stress, $\sigma_{f}$. The drop is not instantaneous; over the time during which friction drops, the plates slip a distance, $D_{c}$, as illustrated in the graph at the bottom. When $D_{c}$ is small, the dynamic stress drop, $\Delta \sigma_{d}=\sigma_{0}-\sigma_{f}$, initially drives the sliding. The sliding stops when the shear stress drops below a final frictional stress, $\sigma_{1}$. A variety of mechanisms can stop sliding, such as geometric and compositional heterogeneity, and dynamically changing velocity- or history-dependent friction, and so $\sigma_{1}$ is not necessarily equal to $\sigma_{f}$. The difference between initial and final frictional stresses is the static stress drop, $\Delta \sigma_{s}=\sigma_{0}-\sigma_{1}$.

With seismological methods, one can determine $D, S$, and the slip velocity, $v_{\mathrm{D}}$. Given the rigidity of crustal rocks, $\mu$, and the shear-wave velocity, $\beta$, the dynamic and static stress drops are $\Delta \sigma_{\mathrm{d}}=v_{\mathrm{D}} \mu / \beta$ and $\Delta \sigma_{\mathrm{s}}=\mu D / S^{1 / 2}$. Both stress drops typically range from 1 to $10 \mathrm{MPa}$, although there are exceptions. Seismological methods measure only transient processes, so they determine stress differences, not the values of the physically impor$\operatorname{tant}$ stresses $\sigma_{0}$ and $\sigma_{1}$.

A convenient measure of the overall size of an earthquake is the seismic moment defined by $M_{0}=\mu S D$. The seismic moment is measured in energy units, but it does not directly represent the energy released by an earthquake. The magnitude, $M$, of an earthquake is given in terms of the seismic moment by $M=\left(\log M_{0}-9.1\right) / 1.5$.
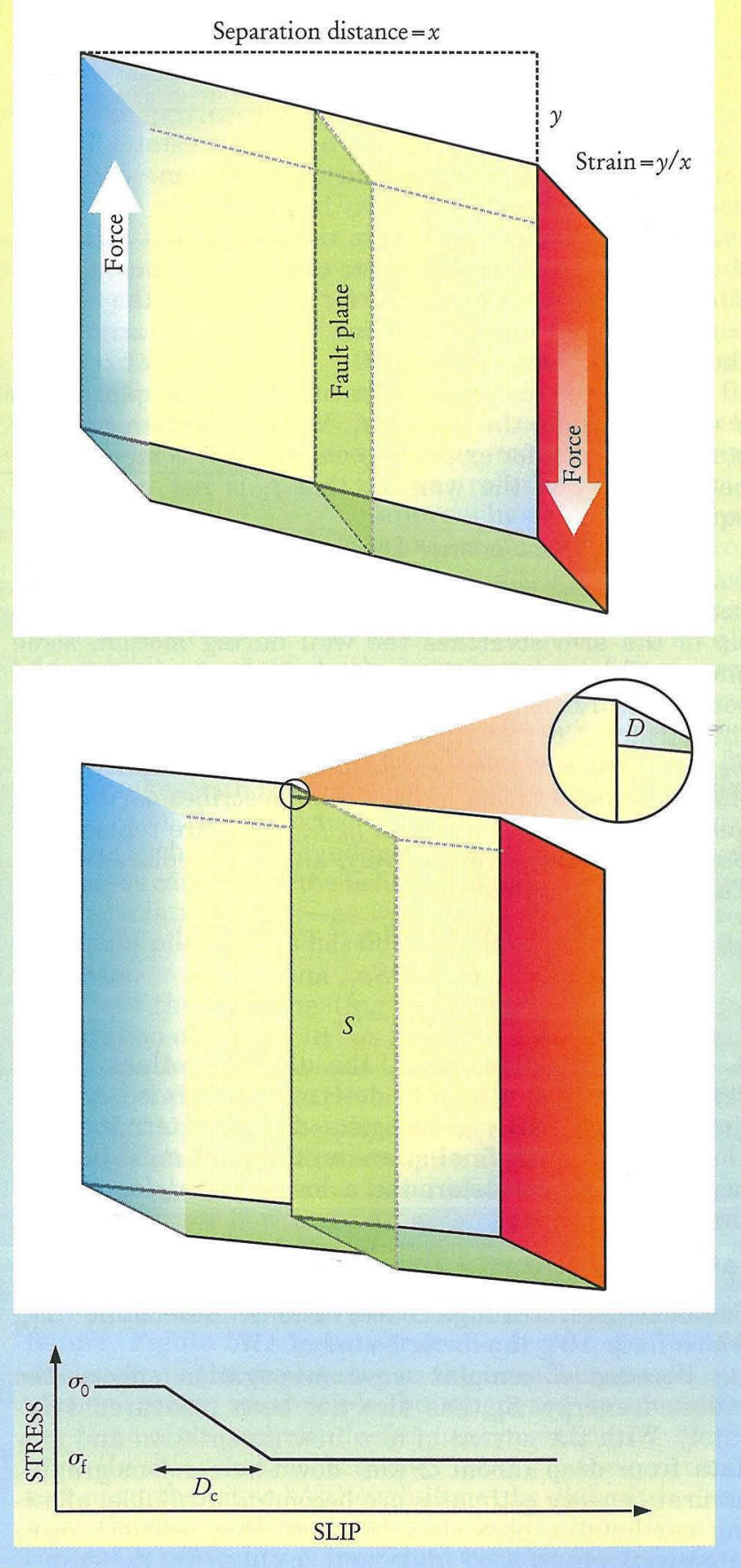

smallest-scale structure, are filtered out because of the difficulty in modeling them. At frequencies greater than $0.5 \mathrm{~Hz}$, the scattering of waves and other seismic complexities produce waveforms that cannot be explained with a simple model. Thus, models typically generate what should be regarded as low-pass filtered rupture patterns; the real slip distribution is probably far more complex with shortwavelength irregularities. Short-period seismic waves seen on seismograms and felt by people are generally believed to be caused by a fractal distribution of small fault-slip zones, each zone radiating short-period waves.

Slip distributions can be obtained at various times while earthquakes are in progress. Slip is typically heterogeneous in time, just as it is in space. By analyzing slip in both space and time, earthquake scientists can gain insight into rupture dynamics.

\section{Rupture dynamics and energy}

The limited resolution of seismic methods prohibits earthquake physicists from determining every detail of rupture patterns on irregular fault planes. The stress-time histories obtained through rupture patterns are therefore complemented by measurements of such integrated quantities as the total radiated energy, $E_{\mathrm{R}}$, and the seismic moment, 
$M_{0}$, which gives the total amount of slip.

Ultimately one needs to consider the energy budget for an extended fault zone, but a relatively simple spring system nicely elucidates how energy is partitioned during an earthquake. The spring is initially stretched a distance, $x_{0}$, from its equilibrium length by a force, $f_{0}$. This corresponds to the state before an earthquake. The stretched spring is held against a wall with static friction that balances $f_{0}$. During an earthquake, some energy is used to fracture rocks; this may be modeled in the spring system by attaching an arm to the spring that scratches the wall. When friction drops to the kinetic value, $f_{\mathrm{f}}$, presumed to happen instantly for simplicity, the spring begins to recoil under a driving force, $f_{0}-f_{\mathrm{f}}$, scratching the wall as it goes.

Eventually the spring stops at $x_{1}$, with a displacement $d=x_{0}-x_{1}$ when the force is $f_{1}$. Various mechanisms can stop the motion, for example geometrical or compositional heterogeneity of the wall, so that $f_{1}$ is not necessarily equal to $f_{\mathrm{f}}$.

The frictional energy loss is $E_{\mathrm{F}}=d f_{\mathrm{f}}$, and the total potential energy change, including strain and gravitational potential energy changes, is $\Delta W=1 / 2\left(f_{0}+f_{1}\right) d$. While the tip of the arm scratches the wall during motion, some energy, $E_{\mathrm{R}}$, is radiated as elastic (seismic) waves, and some fracture energy, $E_{G}$, is spent mechanically damaging the surface. Conservation of energy requires $E_{\mathrm{R}}=\Delta W-$ $E_{\mathrm{F}}-E_{\mathrm{G}}$. The spring system just discussed is analogous to the earthquake model in box 1 and describes earthquake energetics fairly accurately if $f_{0}, f_{1}$, and $f_{\mathrm{f}}$ are replaced by $S \sigma_{0}, S \sigma_{1}$, and $S \sigma_{f}$, respectively, and $d$ is replaced by $D$. Thus, for earthquakes,

$$
\begin{gathered}
\Delta W=D S\left(\sigma_{0}+\sigma_{1}\right) / 2, \\
E_{\mathrm{F}}=D S \sigma_{\mathrm{f}}, \text { and } \\
E_{\mathrm{R}}=\Delta W-E_{\mathrm{F}}-E_{\mathrm{G}} .
\end{gathered}
$$

As discussed in box 1 , the absolute values of the stresses $\sigma_{0}$ and $\sigma_{1}$ cannot be determined. This is a serious limitation, in that seismologists cannot determine $\Delta W$. However, since the final stress on the fault must be nonnegative they can determine a lower bound for $\Delta W$. In that case,

$$
\Delta W=D S\left(\sigma_{0}+\sigma_{1}\right) / 2 \geq D S\left(\sigma_{0}-\sigma_{1}\right) / 2=D S \Delta \sigma_{\mathrm{s}} / 2 \equiv \Delta W_{0} .
$$

Seismologists, through observations, determine $\Delta \sigma_{s}$, which fixes $\Delta W_{0}$, the lower bound of $\Delta W$.

Because of complex wave propagation effects, the radiated energy, $E_{\mathrm{R}}$, has also not been measured accurately. With the advent of new instrumentation and new data from deep (about $2 \mathrm{~km}$ ) down-hole seismographs, accurate energy estimates are becoming available, allowing earthquake physicists to study this problem more quantitatively.

A comparison of $E_{\mathrm{R}}$ and $\Delta W_{0}$ measured for the 1994 deep Bolivian earthquake $(M=8.3)$ yields an interesting result $^{5}$ (also see PHYSICS TODAY, October 1994, page 17). This earthquake, the largest deep-focus earthquake ever recorded, occurred at a depth of $635 \mathrm{~km}$. The comparison shows that $\Delta W_{0}=1.4 \times 10^{18} \mathrm{~J}$ and $E_{\mathrm{R}}=5 \times 10^{16} \mathrm{~J}$, which is only $3 \%$ of $\Delta W_{0}$. The difference, $\Delta W_{0}-E_{\mathrm{R}}=1.35 \times 10^{18} \mathrm{~J}$, was not radiated, and must have been deposited near the focal region, probably in the form of thermal energy. That energy, about $10^{18} \mathrm{~J}$, is comparable to the total thermal energy released during large volcanic eruptions such as Mount Saint Helens in 1980. Moreover, the thermal energy must have been released in a relatively small area, about $50 \times 50 \mathrm{~km}^{2}$, within a time span of about 1 minute. The
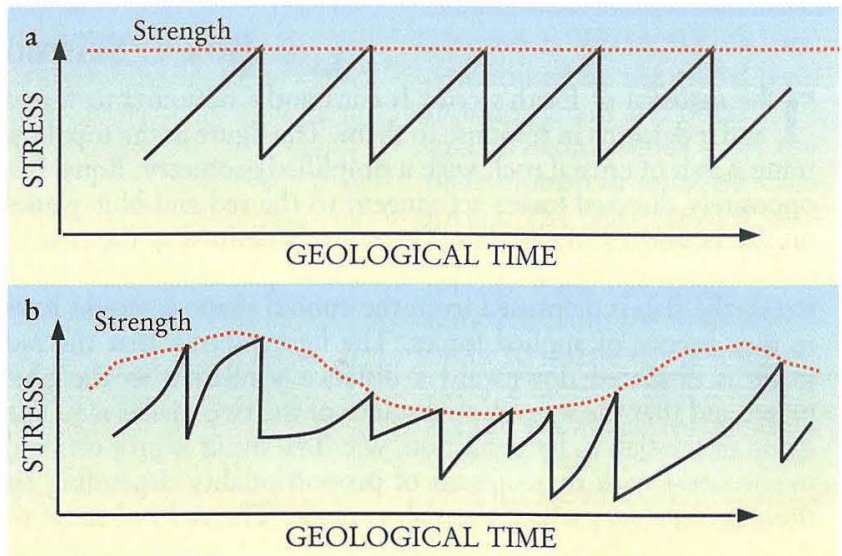

Figure 2. DEVELOPMENT OF EARTHQUAKES, shown schematically. (a) The spatially averaged stress on a fault, varying over geological time. Tectonic stress slowly builds up along a fault until it reaches the local strength, defined as the critical stress necessary for failure (dashed line), and then an earthquake occurs with a sudden stress drop. A new earthquake cycle subsequently begins. (b) A more realistic version of the process includes the complications of variable strength, loading rate, and stress drop.

mechanical part of the process, the seismic waves accompanying the earthquake, was only a small component. Thus, the Bolivian earthquake was more of a thermal event than a mechanical one.

With such a large quantity of nonradiated energy, the temperature in the focal region of the Bolivian earthquake must have risen significantly. The actual temperature rise, $\Delta T$, depends on the thickness of the fault zone, which is not known, but for a zone whose thickness is only a few centimeters, the temperature could have risen to above $5000^{\circ} \mathrm{C}$.

\section{Shallow earthquakes and many 'if's}

Although the pressure-temperature environment for shallow earthquakes may be different from that for deep earthquakes, a simple calculation shows that if $\sigma_{f}$ is comparable to $\Delta \sigma_{\mathrm{s}}$ and $\Delta \sigma_{\mathrm{d}}$, about $10 \mathrm{MPa}$, then the effect of shear heating is significant. If the thermal energy is contained within a zone a few centimeters thick around the slip plane during seismic slip, the temperature can easily rise to $100-1000^{\circ} \mathrm{C}$. If a fault zone is dry, melting may occur and friction may drop. If some fluid exists in that zone, the thermal energy will expand the fluid, which could reduce the normal stress on points of contact between the two fault planes and thus reduce the frictional stress. The key question is how thick the fault slip zone is. Geologists have examined many exhumed faults. Some of them have a very thin (about $1-\mathrm{mm}$ ) distinct region where fault slips seem to have occurred repeatedly. ${ }^{6}$ In other cases, several thin slip zones were found, but evidence shows that each represents a distinct earthquake. Thus, geological evidence suggests a thin slip zone, at least for some faults, but the question remains debatable.

The energy budget of earthquakes provides more insight into the stresses occurring in fault zones. Figure 4 shows the observed ratio, $E_{\mathrm{R}} / M_{0}$, which specifies how much energy is radiated from a unit fault area per unit 

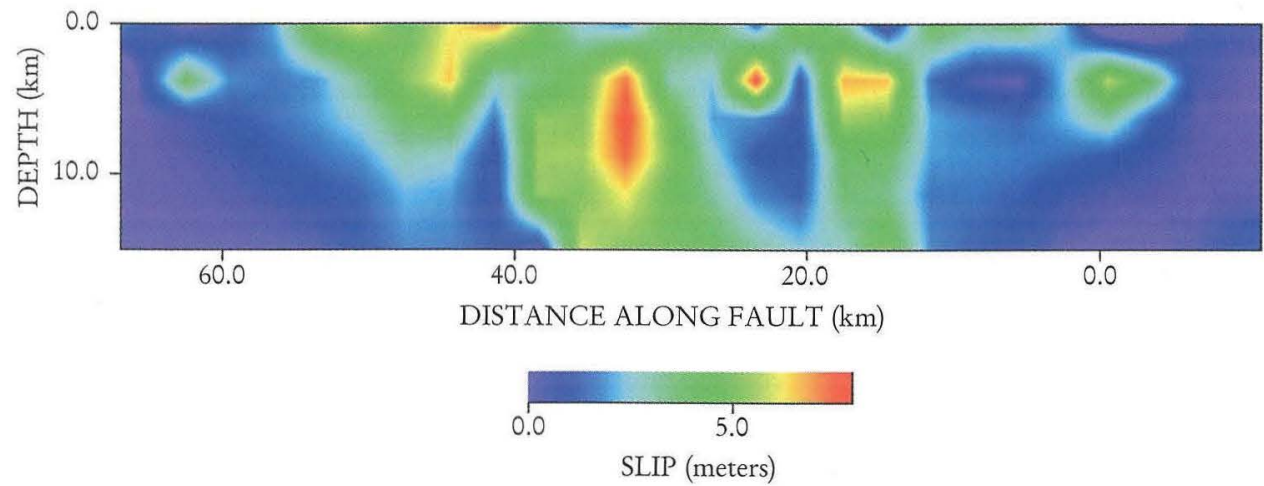

FIGURE 3. SLIP DISTRIBUTION on the fault plane of the (magnitude 7.3) 1992 Landers, California, earthquake. The origin for distance measurements is the epicenter, the point on the surface just above the starting point of rupture. The largest slip, nearly $7 \mathrm{~m}$, occurred underground about $33 \mathrm{~km}$ from the epicenter. (Adapted from ref. 4.)

slip. Using the definition of $M_{0}$ given in box 1 , one can write

$$
E_{\mathrm{R}} / M_{0}=\frac{E_{\mathrm{R}}}{\mu S D}=\frac{1}{\mu} \frac{\left(E_{\mathrm{R}} / S\right)}{D}
$$

Although the measurement errors are still large, figure 4 indicates that the ratio for large earthquakes $(M>5)$ is 10-100 times larger than that for small events $(M<2.5)$. The increase in the ratio suggests some drastic change in fault dynamics between small and large earthquakes. ${ }^{7}$ Box 2 on page 39 describes some possible mechanisms for such a change.

\section{Earthquakes as complex systems}

Large-magnitude earthquakes are rare events. To a very good approximation, the rate of occurrence of earthquakes falls exponentially as a function of magnitude, as is shown in figure 5a. This exponential fall is called the Gutenberg-Richter relation. Several mathematical models that reproduce this relation have been proposed, including a mechanical slider-block system, ${ }^{8}$ a percolation model, ${ }^{9}$ and a sand-pile model. ${ }^{10}$ Here we consider a one-dimensional branching model, essentially a percolation model. We numerically model a seismic fault as a distribution of many small patches, or areas; if one patch fails, it can trigger failures in patches at $s$ nearby sites with a transition probability $p$. The product $e=p s$ is the expectancy of the number of failed patches at each step.

If $e<1$, then the growth of the failing region will

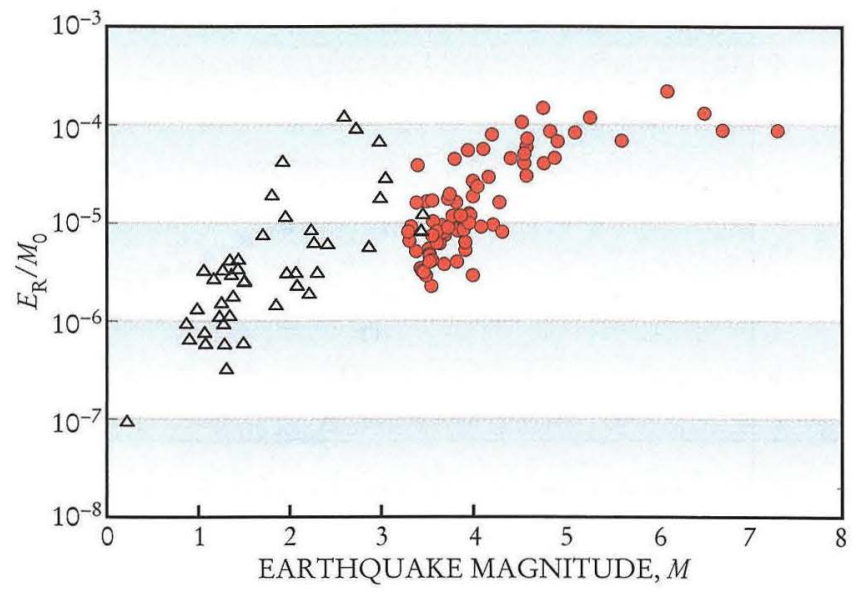

eventually stop when a total of $F$ patches has failed. The whole process corresponds to an earthquake rupture. If the simulation is repeated many times, we find a power-lawlike relation between $\log F$ and the number of cases, $n$, in which at least $F$ patches failed. Figure $5 \mathrm{~b}$ shows the results of numerical simulations performed for $s=10$ and three values of the expectancy, $e=0.8,0.9$, and 0.99. As the expectancy approaches 1 , the plots of figure $5 \mathrm{~b}$ tend to linearity, corresponding to the GutenbergRichter relation. But also, as $e$ approaches 1 , there is occasional runaway triggering, which would indicate an exceptionally large earthquake violating the Gutenberg-Richter relation. ${ }^{11}$ Certain mature faults, such as the San Andreas fault in California, do exhibit an anomalously large number of very-largemagnitude earthquakes. There have been more earthquakes with $M \approx 8$ on the San Andreas fault than predicted by the Gutenberg-Richter relation and relatively few earthquakes with magnitudes between 6 and 7. This deviation from the expected relation suggests a runaway process such as that found in the model. Thus, earthquake processes can exhibit the behavior of complex systems, and the percolation model-as well as the slider-block system and sand-pile model-has built-in features that mimic this behavior.

Both the shear heating and the elastohydrodynamic lubrication discussed in box 2 tend to promote slip motion as the earthquake becomes larger, that is, the slip growth rate depends nonlinearly on the slip itself. Lubrication may thus be one of the physical mechanisms that causes earthquakes to behave as complex systems.

For long-term seismic hazard assessment, it would be useful to know how large an area of the fault is close to failure; the size of the earthquake will ultimately be determined by the size of this critical area. It would also be helpful to know just how close the critical area is to failure. Figure 5b suggests that the degree of criticality, analogous to the expectancy in the branching-model simulation, could, in principle, be diagnosed by measuring the slope of the magnitude-frequency relationship for the area. (In this context, "frequency" refers to the occurrence rate of earthquakes.) Although it may be difficult to determine the degree of criticality with seismic data alone, the concept of criticality is important because it suggests the use of other methods to monitor the state of the crust. For example, electromagnetic methods could be used to moni-
FIGURE 4. THE RATIO of the radiated energy $E_{\mathrm{R}}$ to the seismic moment $M_{0}$, plotted versus magnitude. The data for large earthquakes (filled circles) are from broadband networks in southern California and those for small earthquakes (triangles) are from a down-hole seismograph. ${ }^{14}$ The increase of the ratio with magnitude suggests a dramatic change in fault dynamics between small and large earthquakes. (Adapted from ref. 7.) 


\section{Box 2. Fault Lubrication}

$\mathrm{T}$ he striking feature of figure 4 is that it indicates that large earthquakes have a substantially greater radiated energy per unit fault area per unit slip than do small earthquakes. One possible explanation of this phenomenon is that large earthquakes typically occur on well-developed, through-going faults and do not use much energy making new surfaces. Small earthquakes, on the other hand, often occur on small cracks and expend a lot of energy creating the new surfaces needed for the crack to grow, leaving less energy to be radiated.

We suggest another mechanism responsible for the different dynamics between large and small earthquakes based on the idea of fault lubrication. Suppose the thickness of the slip zone, $w$, is small and does not depend on the event size. The total thermal energy produced during fault slipping is $E_{\mathrm{F}}=\sigma_{\mathrm{f}} D S$; if this energy is distributed over a volume of $S w$, the temperature rise, $\Delta T$, is proportional to $E_{\mathrm{F}} / S w=\sigma_{\mathrm{f}} D / w$. Thus, as $D$ increases with the earthquake magnitude $M$, the temperature rises significantly, friction is reduced, and fault motion occurs more rapidly, thereby radiating more energy.

In the presence of fluids, a strictly mechanical effect-elastohydrodynamic lubrication-can reduce friction independent of the previously described thermal effect. If the fault zone is thin and rough, and if the material in the fault zone behaves like a viscous fluid, the shearing of the fault fluid produces a viscous stress that is balanced by dynamic pressure. The fluid pressure rises, which both reduces friction and elastically deforms the fault planes. Such elastohydrodynamic lubrication also reduces friction for large events relative to small ones. ${ }^{12}$

Thus, when the slip and slip velocity are large and the fault zone is thin, a pressure increase, caused by either thermal or hydrodynamic effects, becomes large enough to smooth out the

tor fluid flow in the crust. When fluid migrates in the crust and weakens some parts of fault zones, the crust could approach a critical state and produce electrical or magnetic noise. If the crust has a low degree of criticality, a small perturbation in stress or weakness is not very likely to cause a large event.

\section{Connecting small and large scales}

A number of approaches link observations with mechanics in earthquake physics. The fundamental problem is to understand the microscopic processes using macroscopically observed parameters. We who study earthquakes, like those who established statistical mechanics, must

FIGURE 5. EARTHQUAKE SIZE DISTRIBUTIONS.

(a) Magnitude-frequency relationship for earthquakes worldwide, computed from various catalogs that cover several periods between 1904 and 2000. The $y$-axis gives the average number of earthquakes per year $(N)$ with a magnitude of at least $M$. The solid line gives the best fit to the Gutenberg-Richter relation, $\log N=a-b M$. Note that the slope is approximately -1 and that, on average, about one earthquake with $M \geq 8$ occurs every year. (b) The results of numerical branching-model simulations in which a given failed patch triggered new failures in an average of $e$ neighboring patches. The graph plots the num-

ber of simulations $(n)$ in which at least $F$ patches failed as a function of $\log F$, for three cases, $e=0.8,0.9$, and 0.99 . Note the change in the slope for the initial part of the curves as $e$ increases from 0.8 to 0.99 . Because the $y$-axis is an integrated variable accounting for all trials in which at least $F$ sites failed, trials with anomalously high failures are not readily discernible in the figure. They are, however, evident in reference 11. irregularities on the fault surface by elastic deformation. This smoothing suppresses short-period ground motion caused by the fault surfaces rubbing against each other. During the 1999 Chi-Chi, Taiwan, earthquake of magnitude $M=7.6$, the observed ground-motion velocity near the northern end of the fault was extremely large (about $3 \mathrm{~m} / \mathrm{s}$-the largest ever recorded), but short-period acceleration was not particularly strong, so the damage to ordinary structures by shaking was minor. ${ }^{13}$ The counterintuitive coupling of large ground-motion velocity with minor structural damage could be a manifestation of lubrication effects. However, since the Chi-Chi earthquake is the only one for which such large slip and slip velocity have been instrumentally observed, whether the counterintuitive coupling represents general behavior remains to be seen.

Some caveats are in order. Whether lubrication occurs depends on many factors, such as the effective permeability in the fault zone, compressibility of fault rocks, and the viscosity of fault fluid. Although the idea of fault lubrication seems promising, it is fairly new and has not yet stood the test of time. In this paradigm, the thin slip zone is the key concept. If some mechanisms exist to distribute energy over a thick zone (greater than $10 \mathrm{~cm}$ ) during a seismic event, it may be possible to maintain high friction on a fault plane. If the viscosity of the frictional melt is high, melting may actually increase the fault friction. Although figure 4 indicates to us that the fault dynamics of large and small earthquakes are quite different, one could argue that the seismological data presented are not that robust. Thus, debate, controversy, and speculation are likely to continue for some time. However, in view of the large slip and slip velocity associated with seismic faulting, we believe significant lubrication is likely to occur if the slip zone is thin.
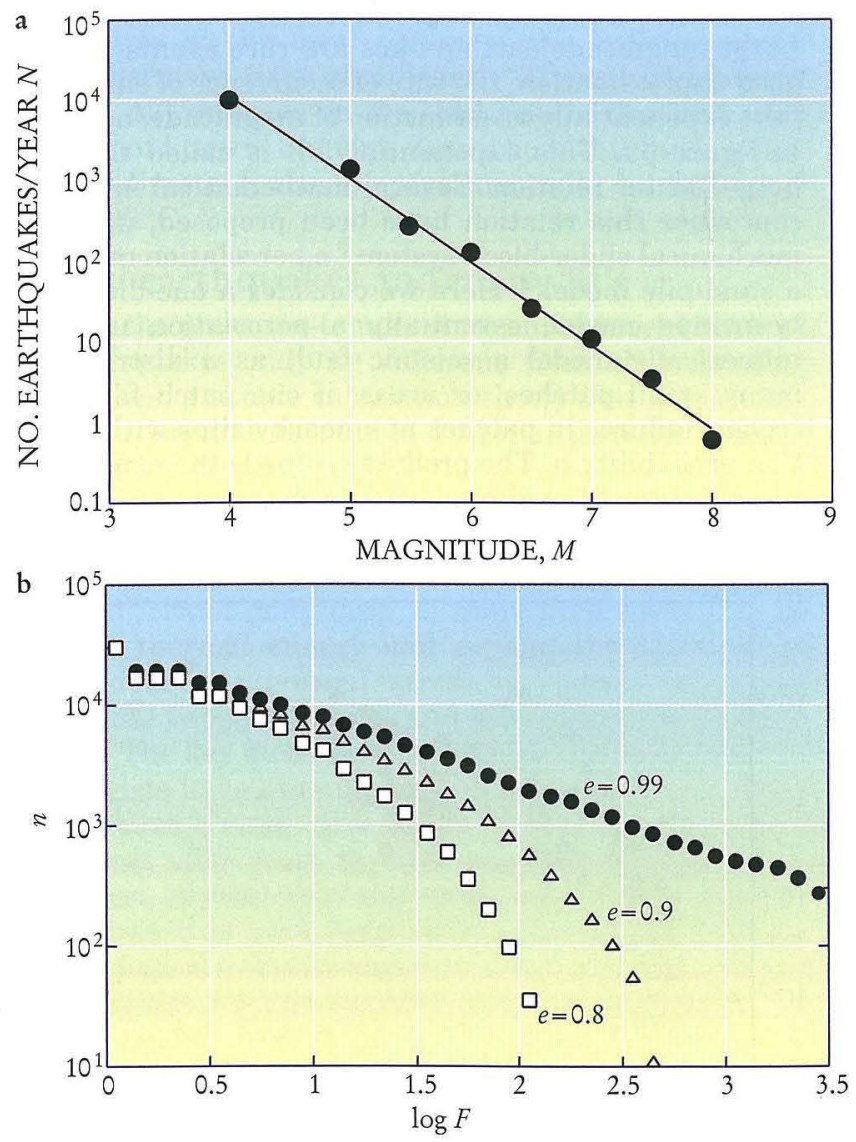
develop methods to connect radically different size scales.

One microscopic feature is of particular interest to us: the thin fault zone. During large seismic events, thermal and mechanical processes may result in low friction in such zones. This low friction, combined with estimated static and dynamic stress drops, suggests that mature seismic fault systems operate at relatively low stresses, on the order of tens of $\mathrm{MPa}$. On the other hand, the crust supports large surface loads, such as mountains, that require its strength to be at least $100 \mathrm{MPa}$. This means that the stress in the crust is spatially extremely heterogeneous, and the system organizes itself into a somewhat precarious state with low stress on major faults.

But what happens during an earthquake? In the selforganized system just described, a small stress perturbation can trigger a seismic event. The growth of the earthquake is controlled by heterogeneous friction on the fault, which in turn may be affected by nonlinear processes involved in lubricated dynamic slip. This growth process results in the standard magnitude-frequency relationship with occasional unusually large earthquakes, possibly caused by large runaway events. Elastohydrodynamic lubrication in a thin fault zone may change the roughness of the fault plane and thereby change the frequency spectrum of ground motion. Strategies for designing structures that will withstand ground motions from large earthquakes would have to account for this change in frequency spectrum. The lubrication model we have proposed is new, and debate continues about the microscopic processes occurring in the fault zone. Understanding these processes is key to a better understanding of seismicity, rupture dynamics, and ground motion characteristics, which will lead to effective seismic risk mitigation measures.

\section{References}

1. Some good general references are: K. Aki, P. G. Richards, Quantitative Seismology, Freeman, San Francisco (1980); B. A. Bolt, Earthquakes, Freeman, New York (1999); T. Lay, T. C. Wallace, Modern Global Seismology, Academic Press, New York (1995); C. H. Scholz, The Mechanics of Earthquakes and Faulting, Cambridge U. Press, New York (1990).

2. C. Tsuboi, Bull. Earthquake Res. Inst., U. Tokyo 10, 411 (1932).

3. E. Rabinowicz, Friction and Wear of Materials, Wiley, New York (1995).

4. D. J. Wald, T. H. Heaton, Bull. Seismol. Soc. Am. 84, 668 (1994).

5. H. Kanamori, T. H. Anderson, T. H. Heaton, Science 279, 839 (1998).

6. F. M. Chester, J. S. Chester, Tectonophysics 295, 199 (1998).

7. H. Kanamori, T. Heaton, in GeoComplexity and the Physics of Earthquakes, J. R. Rundle, D. L. Turcotte, W. Klein, eds., Geophysical monograph no. 120, American Geophysical Union, Washington, DC (2000), p. 147.

8. R. Burridge, L. Knopoff, Bull. Seismol. Soc. Am. 57, 341 (1967).

9. M. Otsuka, Zisin 24, 215 (1971).

10. P. Bak, C. Tang, J. Geophys. Res. 94, 15635 (1989).

11. H. Kanamori, J. Mori, in Problems in Geophysics for the New Millennium: A Collection of Papers in Honor of Adam $M$. Dziewonski, E. Bochi, G. Ekström, A. Morelli eds., Compositori, Bologna, Italy (2000), p. 73. See, in particular, fig. 9.

12. E. E. Brodsky, H. Kanamori, J. Geophys. Res. (in press).

13. K-F. Ma, C-T. Lee, Y-B. Tsai, T. C. Shin, J. Mori, EOS Transactions 80, 605 (1999).

14. R. Abercrombie, J. Geophys. Res. 100, 24015 (1995). 